\title{
Predicción de la dureza de la aleacion Zn-Al-Cu de acuerdo a su composición en peso
}

\author{
Prediction of Hardness of the $\mathrm{Zn}-\mathrm{Al}-\mathrm{Cu}$ \\ Alloys of Agreement by Composition in Weight
}

\author{
Villegas-Cárdenas José David \\ Universidad Politécnica del Valle de México \\ Tultitlan, Estado de México \\ Correo:jdvc76@yahoo.com.mx \\ Camarillo-Villegas Alejandra \\ Universidad Politécnica del Valle de México \\ Tultitlan, Estado de México \\ Correo:v_c_a_77@hotmail.com \\ Juanico-Lorán Antonio \\ Universidad Politécnica del Valle de México \\ Tultitlan, Estado de México \\ Correo:ajuanico@yahoo.com.mx
}

\author{
Espinosa-Rojas Raúl \\ Universidad Autónoma Metropolitana \\ Azcapotzalco, México \\ Correo:rer21@hotmail.com
Camacho-Olguín Carlos
Universidad Politécnica del Valle de México
Tultitlan, Estado de México
Correo:ccamacho@upvm.edu.mx

Información del artículo: recibido: febrero de 2011, reevaluado: noviembre de 2011 y febrero de 2012, aceptado: septiembre de 2012

\section{Resumen}

Se desarrollaron diez aleaciones $\mathrm{Zn}-\mathrm{Al}$ - $\mathrm{Cu}$ divididas en dos partes, de acuerdo a dos zonas presentadas en los diagramas isopléticos de Hans (Villas et al., 1995). Se incrementó el porcentaje de Cu y Al paulatinamente. Posteriormente se desarrollaron pruebas de macrodureza y de ese análisis se obtuvieron dos ecuaciones que permiten pronosticar y diseñar aleaciones de una dureza determinada de acuerdo a su porcentaje en peso de cada elemento, con un error menor que 5\%. Como se demuestra en este trabajo, con estas ecuaciones es posible desarrollar aleaciones sustitutas base aluminio por una aleación base zinc o viceversa, teniendo la misma dureza para cada tipo de aleación y eliminando el problema del cambio volumétrico debido a la presencia de la fase $\varepsilon$.

\section{Descriptores:}

- dureza

- fase

- dendríta

- energía de Gibbs 


\begin{abstract}
Ten alloys $\mathrm{Zn}-\mathrm{Al}-\mathrm{Cu}$ were developed in two parts, in agreement to two zones presented in the isopleth diagrams (Villas et al., 1995). The percentage of $C u$ and $A l$ was systematically varied. Subsequently, hardness measurements were performed. These measurements allowed establishing two equations that predict the hardness with an error lower than $5 \%$. With these equations, it is possible to obtain alloys that replace Al base alloys by a $\mathrm{Zn}$ base alloy, having the same hardness. This implicates also the elimination of the volumetric change in the presence of $\varepsilon$ phase.
\end{abstract}

Keywords:

- hardness

- phase

- dendritic

- Gibbs energy

- percent by weight, Zn-Al-Cu

\section{Introducción}

En la última década, las aleaciones de $\mathrm{Al}$ han sido, después del acero, las más utilizadas a nivel mundial. Algunas de éstas se usan para fabricar cubiertas protectoras de vapor, agua marina, engranes, bujes y otros materiales expuestos a fricción. También son de gran interés industrial y de investigación. Algunas aleaciones binarias ricas en zinc, con alto contenido de aluminio, modificadas con pequeñas cantidades de elementos de aleación $(\mathrm{Cu}, \mathrm{Mg}$ y/o $\mathrm{Si})$, presentan propiedades interesantes. Por ejemplo, el Zn-Al tiene propiedades similares a las de ciertos bronces, que son empleados para bujes sujetos a la abrasión, además estas aleaciones pueden elaborarse por medio de distintos procesos, lo que las hace aún más atractivas para su estudio (Aragón et al., 2007; Ahmed, 2006; Mohammed, 2011; De Micco et al., 2007; Chen et al., 2004).

No obstante, los inconvenientes que presentan estas aleaciones son, por un lado, la inestabilidad dimensional (Temel y Yasin, 2009) debido a la transformación de la fase metaestable $\varepsilon\left(\mathrm{CuZn}_{4}\right)$, dentro de la fase estable $\tau^{\prime}$ por la reacción de las cuatro fases $\left(\alpha+\varepsilon \rightarrow \tau^{\prime}+\eta\right)$, lo cual origina una expansión irreversible de 4 a $5 \%$. Recientemente se ha pretendido cambiar la fase $\varepsilon$ por la fase $\theta$ y de esta forma eliminar el problema de la expansión volumétrica, aunque esto tendría un cierto costo en la densidad de la aleación debido al aumento de la cantidad de Zn.

Sin embargo, la República Mexicana cuenta con abundantes recursos para producir zinc, de ahí que uno de los objetivos de este trabajo es obtener aleaciones base $\mathrm{Zn}$, capaces de poder sustituir las aleaciones base Al con las mismas propiedades mecánicas, en particular la macrodureza. Por otra parte, otro de los objetivos es obtener una serie de ecuaciones que pronostiquen de acuerdo a su porcentaje en peso de cada elemento la macrodureza.

\section{Desarrollo teórico}

La base para la selección de las aleaciones utilizadas fue de acuerdo a dos criterios, el primero fue tener siempre en una misma zona cada una de las series, y el segundo que una de las series fuese rica en $\mathrm{Zn}$, en la mayoría de sus muestras y la otra en Al. Para cumplir con el primer criterio se utilizaron 5 diagramas isopléticos de Hans (Villas et al., 1995), escogiendo dos zonas compuestas por las fases $(\eta-\tau-\alpha)$, la cual tiene un alto contenido de $\mathrm{Al}$ y la zona $(\varepsilon-\alpha)$ que tiene un alto contenido de $\mathrm{Zn}$. La figura 1 muestra la zona compuesta por las fases $(\eta-\tau-\alpha)$ de forma achurada, mientras que la zona sombreada de color negro está conformada por las fases $(\varepsilon-\alpha)$.

Las aleaciones que se eligieron, de acuerdo a los criterios antes mencionados, se pueden ver en dos líneas totalmente rectas, como se muestra en el diagrama ternario en la figura 2.

Cada una de las series utilizadas puede modelarse por medio de una regresión lineal y obtener las siguientes ecuaciones para cada una:

$\mathrm{Zn}_{(\eta-\tau-\alpha)}=-1.3706\left(\mathrm{Cu}_{(\mathrm{p})}\right)+0.7067$

$\mathrm{Zn}_{(\varepsilon-\alpha)}=-1.8563\left(\mathrm{Cu}_{(\mathrm{p})}\right)+0.9888$

Donde $\mathrm{Zn}_{(\eta-\tau-\alpha)} \mathrm{y} \mathrm{Zn}_{(\varepsilon-\alpha)}$ es la fracción en peso de zinc y $\mathrm{Cu}_{(\mathrm{p})}$ es el porciento en peso de cobre. En las tablas 1 y 2 se muestran los porcentajes en peso de cada una de estas aleaciones.

\section{Desarrollo experimental}

Las aleaciones se desarrollaron por colada convencional y posteriormente se colocaron dentro de un horno convencional a $360^{\circ} \mathrm{C}$, durante 240 horas, con el fin de asegurar la eliminación de la estructura dendrítica. Una vez realizado el proceso de homogenización se realizó un análisis metalográfico convencional de las muestras 


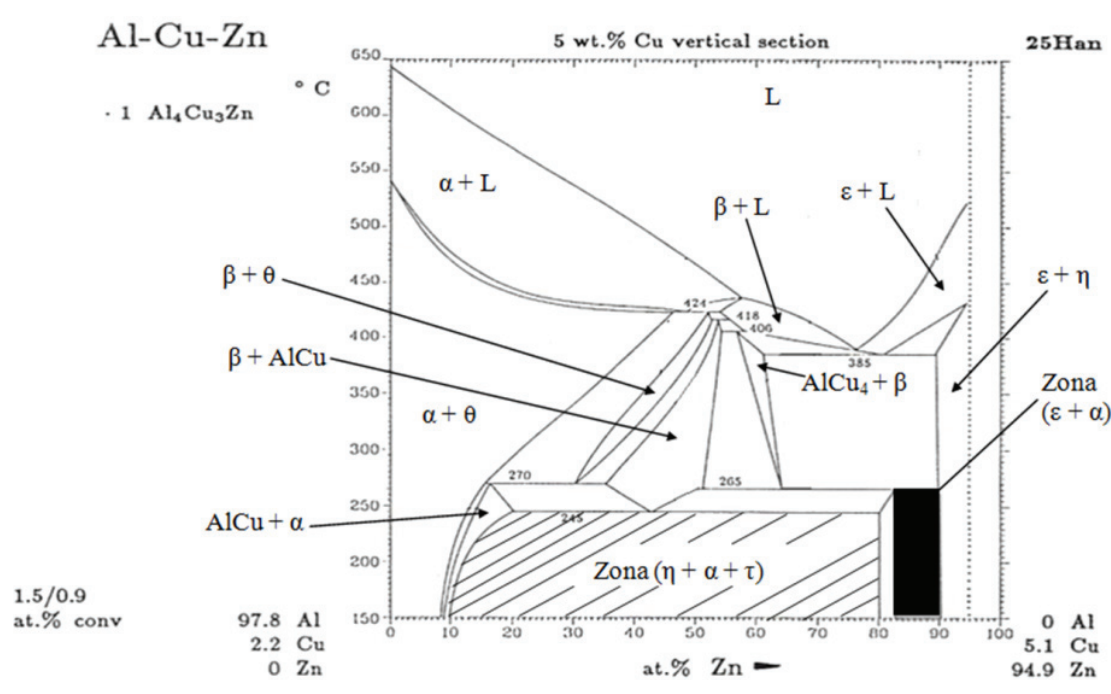

Figura 1. El diagrama isopléticoZn-Al con $5 \%$ de Cu muestra la zona compuesta por las fases $(\eta-\tau-\alpha)$ y de color obscuro la zona compuesta por $(\varepsilon-\alpha)$ (Villas et al., 1995)

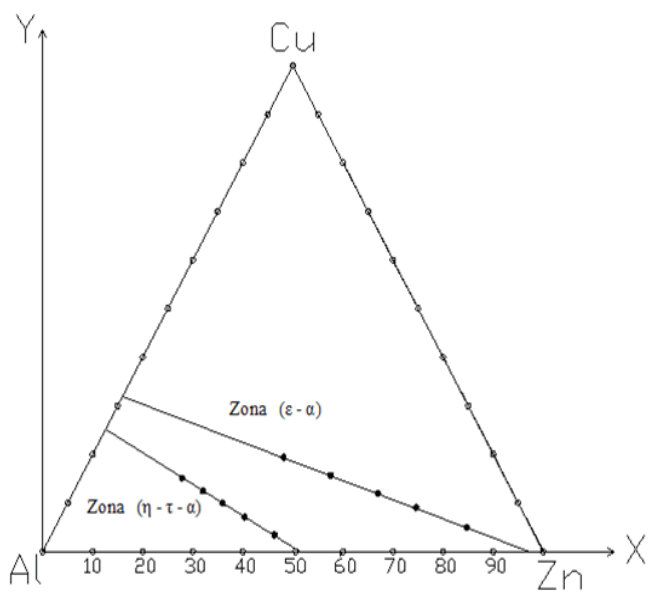

para observar la eliminación de la estructura de colada. La dureza de las muestras, se obtuvo mediante el uso de un macrodurómetro en la escala Rockwell B.

El hecho de modelar el porcentaje en peso de cada elemento químico de las aleaciones, por medio de dos ecuaciones, permite relacionar a éstas con los resultados de dureza.

Tabla 1. Porcentaje en peso de cada una de las aleaciones de la zona compuesta por las fases $(\eta-\tau-\alpha)$

\begin{tabular}{rlll}
\hline \multicolumn{4}{c}{ \% en peso } \\
\hline Muestra & Zn & Al & Cu \\
\hline M1 & 0.64 & 0.31 & 0.05 \\
M2 & 0.57 & 0.33 & 0.10 \\
M3 & 0.50 & 0.35 & 0.15 \\
M4 & 0.43 & 0.37 & 0.20 \\
M5 & 0.37 & 0.38 & 0.25
\end{tabular}

Figura 2. Diagrama ternario de la aleación $\mathrm{Zn}-\mathrm{Al}-\mathrm{Cu}$, se puede observar la linealidad de las aleaciones que fueron utilizadas en este trabajo para cada una de las zonas

Tabla 2. Porcentaje en peso de cada una de las aleaciones de la zona compuesta por las fases $(\varepsilon-\alpha)$

\begin{tabular}{cccc}
\hline \multicolumn{4}{c}{ \% en peso } \\
\hline Muestra & Zn & Al & Cu \\
\hline M6 & 0.90 & 0.05 & 0.05 \\
M7 & 0.80 & 0.10 & 0.10 \\
M8 & 0.72 & 0.13 & 0.15 \\
M9 & 0.62 & 0.18 & 0.20 \\
M10 & 0.52 & 0.23 & 0.25 \\
\hline
\end{tabular}

\section{Discusión y análisis de resultados}

Las figuras 3a y 3b, muestran la probeta M3 en estado de colada y después del proceso de homogeneizado, en estas metalografías se puede apreciar cómo desaparece la estructura dendrítica. 

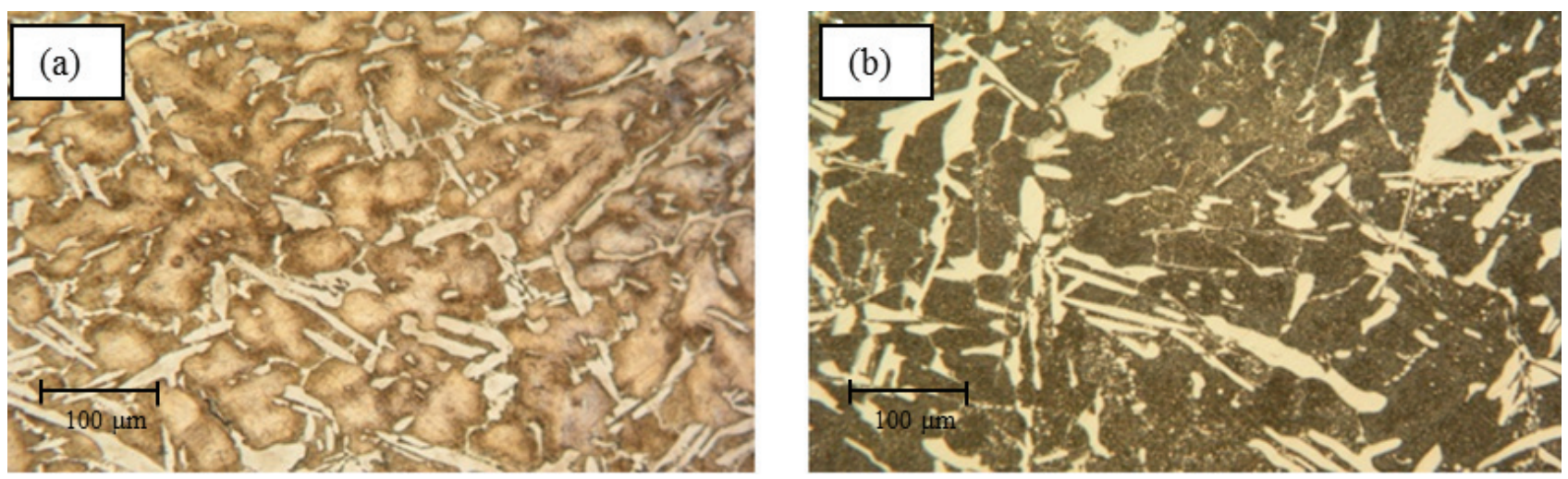

Figura 3. Metalografía de la muestra M3 a 200 aumentos: a) Con estructura dendrítica, b) Después de $240 \mathrm{~h}$ en el horno a $360^{\circ} \mathrm{C}$, donde se puede apreciar la ausencia de la estructura dendrítica

En la figura 4 se muestra el gráfico de la dureza de la serie $(\eta-\tau-\alpha)$ vs el porcentaje en peso de $\mathrm{Cu}$ en forma logarítmica. Las razones para emplear la función logarítmica son primero, obtener una regresión lineal que se ajustará mejor a los resultados y segundo, debido a que no es posible matemáticamente tener un logaritmo 0 , esta función se ajusta mejor a la realidad de una aleación ternaría, en la cual sería imposible tener cero por ciento en uno de los tres elementos, de otra forma sería binaria. La tabla 3 muestra las mediciones de macrodureza en Rockwell B.

De la gráfica de la figura 4 se obtiene una ecuación lineal por medio de una regresión lineal:

$$
\operatorname{Hd}_{(\text {zona }(\eta-\tau-\alpha))}=10.776 \ln \left(\mathrm{Cu}_{(\mathrm{p})}\right)+97.993
$$

donde

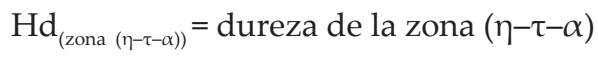

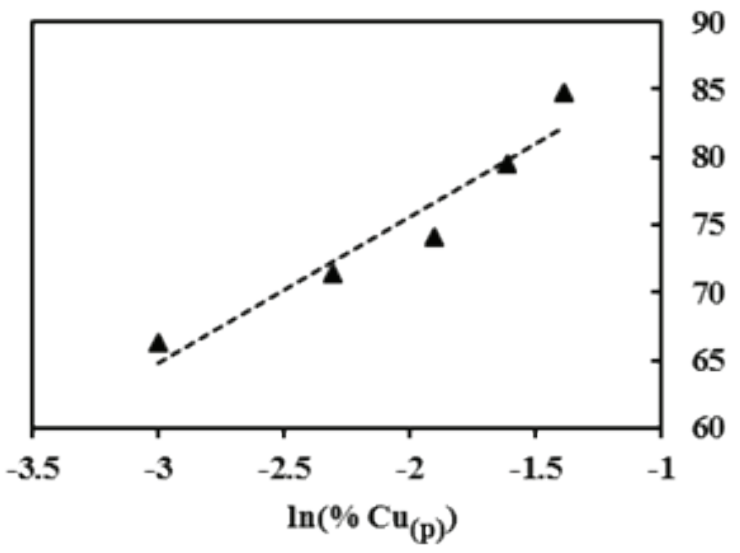

Figura 4. Gráfica de dureza vs porcentaje de $\ln \left(\% \mathrm{Cu}_{(\mathrm{p})}\right)$ para la zona $(\eta-\tau-\alpha)$
$\mathrm{Cu}_{(\mathrm{p})}=$ Fracción en peso de $\mathrm{Cu}$.

Con este modelo se puede pronosticar la dureza de acuerdo al porcentaje en peso de cada uno de los elementos.

Para observar la efectividad de las ecuaciones 1 y 3 se hicieron dos pruebas a 2 y $0.5 \%$ de $\mathrm{Cu}_{(\mathrm{p})}$. Se obtuvieron los porcentajes de cada elemento y con la ecuación (1):

Prueba 1

$\mathrm{Zn}_{(\eta-\tau-\alpha)}=-1.3706(0.02)+0.7067$

$\mathrm{Zn}_{(\eta-\tau-\alpha)}=0.679$

$\mathrm{Al}_{(\eta-\tau-\alpha)}=1-0.02+0.679=0.301$

Prueba 2

$\mathrm{Zn}_{(\eta-\tau-\alpha)}=-1.3706(0.005)+0.7067$

$\mathrm{Zn}_{(\eta-\tau-\alpha)}=0.7$

$\mathrm{Al}_{(\eta-\tau-\alpha)}=1-0.005+0.7=0.295$

La dureza de cada una de estas pruebas se pronosticó con la ecuación (3):

$\operatorname{Hd}_{(\text {zona }(\eta-\tau-\alpha))}=10.776 \ln (0.02)+97.993=55.84$

Tabla 3. Dureza en Rockwell B, de las muestras M1 a M5, en la zona $(\eta-\tau-\alpha)$

\begin{tabular}{cll}
\hline \multicolumn{3}{c}{ Zona $(\eta-\tau-\alpha)$} \\
\hline Muestra & 1n $(\% \mathbf{C u})$ & Dureza RB \\
\hline M1 & 1.61 & 66.22 \\
M2 & 2.30 & 71.37 \\
M3 & 2.71 & 74.00 \\
M4 & 3.00 & 79.43 \\
M5 & 3.22 & 84.63 \\
\hline
\end{tabular}


Prueba 2

$\operatorname{Hd}_{(\text {zona }(\eta-\tau-\alpha))}=10.776 \ln (0.005)+97.993=40.90$

En la tabla 4 se presentan los resultados experimentales con los resultados teóricos antes obtenidos.

Tabla 4. Comparación de la dureza estimada y la real

\begin{tabular}{cccc}
\hline \% Cu & $\begin{array}{c}\text { Dureza real } \\
\text { (R B) }\end{array}$ & $\begin{array}{c}\text { Dureza estimada } \\
\text { (R B) }\end{array}$ & \% Error \\
\hline 0.5 & 39.62 & 40.90 & 3.13 \\
2 & 54.06 & 55.84 & 3.19 \\
\hline
\end{tabular}

La diferencia entre los valores pronosticados y experimentales es de $3 \%$, por lo que se puede tomar como una buena referencia para obtener la dureza a partir de las ecuaciones (1) y (3).

El rango en el porcentaje de $\mathrm{Cu}$ está limitado por la misma ecuación (3). Debido a que es imposible tener durezas negativas, el porcentaje de $\mathrm{Cu}$ debe ser mayor a $0.01123 \%$. El valor máximo de contenido de Cu se obtiene por la ecuación 1, el cual sería de 51.56\%.

En el caso de la ecuación de dureza de la zona $(\varepsilon-\alpha)$ es

$\operatorname{Hd}_{(\text {zona }(\varepsilon-\alpha))}=25.592 \ln \left(\% \mathrm{Cu}_{(\mathrm{p})}\right)+123.41$

Utilizando la ecuación (4) se comparan las durezas experimentales con las pronosticadas, como se muestra en la figura 5, con una $\mathrm{R}^{2}$ de 0.9537; lo cual muestra que al igual que en el caso anterior, se tiene una ecuación que proporciona un pronóstico con un error menor que $5 \%$.

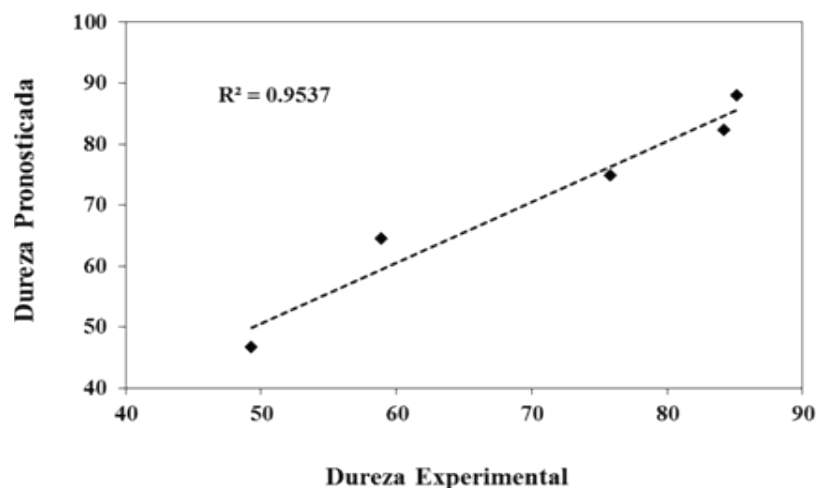

Figura 5. Comparación de la dureza experimental contra el pronóstico obtenido por la ecuación 4

Por último, otra utilidad que tienen las ecuaciones 3 y 4 , es la de obtener la equivalencia de dureza, de acuerdo con el porcentaje de $\mathrm{Al}$ o $\mathrm{Zn}$. Para demostrar esto se tiene por ejemplo la muestra M8 y la muestra M3, que tie- nen casi la misma dureza, pero diferente porcentaje de Al y Zn (tabla 5).

Tabla 5. Comparación de los porcentajes de aluminio con respecto a la dureza experimental

\begin{tabular}{ccccc}
\hline Muestra & $\% \mathbf{Z n}$ & $\% \mathbf{A l}$ & $\mathbf{\%} \mathbf{C u}$ & Dureza (RB) \\
\hline M3 & 50 & 35 & 15 & 74.00 \\
M8 & 72 & 13 & 15 & 72.88 \\
\hline
\end{tabular}

La muestra M8 tiene un mayor contenido de Zn, pero utiliza la tercera parte de Aluminio que la muestra M3, es decir, se sustituye el Al por el Zn.

\section{Conclusiones}

Se demuestra en este trabajo la posibilidad de obtener una serie de ecuaciones (1) y (2), que modelan el porcentaje de cada elemento para cada una de las aleaciones. Es viable obtener otra serie de ecuaciones (3) y (4), que permiten pronosticar la dureza con $5 \%$ de error, con respecto al experimental. Esto permitirá diseñar y crear aleaciones a la medida.

También se demostró que es posible sustituir el Al por el Zn y tener las mismas propiedades mecánicas (dureza), de tal forma que se puedan reducir los costos de algunas aleaciones o darle otras características específicas.

\section{Referencias}

Villars P., Prince A., Okamoto H. Al - Cu - Zn Phase Diagrams,1a ed., Ohio, USA, ASM International, V3, 1995, pp. 3427-3432.

Aragón J.A., Miranda J.R., García Borquez A. Obtención de una microestructura nueva en la aleación $\mathrm{Zn}-40 \%$ at. $\mathrm{Al}-1.5 \%$ at. Cu. Revista Mexicana de Física, volumen 53 (número 3), junio 2007: 149-158.

Ahmed M.M. Corrosion Behaviour of Zn-Al-Cu Alloy in HCl Solution and its Inhibition, Portugaliae Electrochimica Acta, volumen 24 (número 1), enero2006: 1-22.

Mohammed A.A. Uniform and Pitting Corrosion Events Induced by SCN-Anions on Al Alloys Surfaces and the Effect of UV Light. Electrochimica Acta, volumen 56 (número 5), febrero 2011: 2518-2531.

De Micco G., Bohe A.E., Pasquevich D.M. Caracterización de aleaciones $\mathrm{Cu}-\mathrm{Zn}-\mathrm{Al}$ : estabilidad térmica de las fases y decincación. Matéria (Rio J.), volumen 12 (número 2), marzo 2007.

Chen H., Xin X., Dong D.Y., Ren Y.P., Hao S.M. Study on the Stability of T' Phase in the Al-Zn-Cu Ternary System. Acta Metallurgica Sinica, volumen 17 (número 3), junio 2004: 269-273. 
Temel-Savaskan., Yasin-Alemdag. Effect of Nickel Additions on the Mechanical and Sliding Wear Properties of $\mathrm{Al}-40 \mathrm{Zn}-$ 3Cu alloy. Wear, (número 268), octubre 2009: 565-570.

\begin{abstract}
Este artículo se cita:
Citación estilo Chicago

Villegas-Cárdenas, José David, Alejandra Camarillo-Villegas, Antonio Juanico-Lorán, Raúl Espinosa-Rojas, Carlos Camacho-Olguín. Predicción de la dureza de la aleación $\mathrm{Zn}$ - Al-Cu de acuerdo a su composición en peso. Ingeniería Investigación y Tecnología, XIV, 03 (2013): 311-316.

Citación estilo ISO 690

Villegas-Cárdenas J.D., Camarillo-Villegas A., Juanico-Lorán A., Espinosa-Rojas R., Camacho-Olguín C. Prediccion de la dureza de la aleacion Zn-Al-Cu de acuerdo a su composición en peso. Ingeniería Investigación y Tecnología, volumen XIV (número 3), julioseptiembre 2013: 311-316.
\end{abstract}

\section{Semblanza de los autores}

José David Villegas-Cárdenas. Es maestro en ciencias e ingeniería de materiales por la Universidad Autónoma Metropolitana (UAM). Ha sido docente e investigador en la Universidad Politécnica del Valle de México, sus áreas de investigación son manufactura, materiales no ferrosos y modelación.

Alejandra Camarillo-Villegas. Es ingeniera Química por la UAMI y maestra en ciencias e ingeniería, con especialidad en química de los materiales por la UAMA. Fue asesora de las empresas MUEBLA y FOLMEX. Actualmente es profesora e investigadora de la UPVM. Su área de especialización es corrosión de elementos no ferrosos.

José Antonio Juanico-Lorán. Es ingeniero físico por la Universidad Autónoma Metropolitana y maestro en ciencias e ingeniería de materiales con especialidad en física de materiales (nanotecnología) por la UAM. Ha sido docente, investigador y director de laboratorios en la Universidad Autónoma del Estado de Hidalgo y académico en la UAM. Actualmente es profesor-investigador del Grupo de Nanotecnología de la Universidad Politécnica del Valle de México. Áreas de investigación: nanoeducación, estado de la nanotecnología en México, nanoformaciones y nanoerosión por irradiación iónica, difracción de rayos $X$, modelado matemático en nitrurado, síntesis y caracterización de nanoestructuras de carbono.

Raúl Espinosa-Rojas. Es profesor e investigador de la Universidad Autónoma Metropolitana (UAM), miembro del cuerpo de investigación de materiales y especialista en aleaciones no ferrosas.

Carlos Alberto Camacho-Olguín. Ha laborado en empresas como Sosa Texcoco, Nacobre, SpiraxSarco Mexicana y ventas, instalaciones y servicio, con experiencia en ingeniería de procesos, fundición de aleaciones no metálicas, instalación y puesta en marcha de maquinaria y equipos de proceso en empresas químicas, petroquímicas y manufactureras, así como en ventas de equipo de control e instrumentación. Actualmente es profesor investigador de la Universidad Politécnica del Valle de México en el área de nanotecnología. 